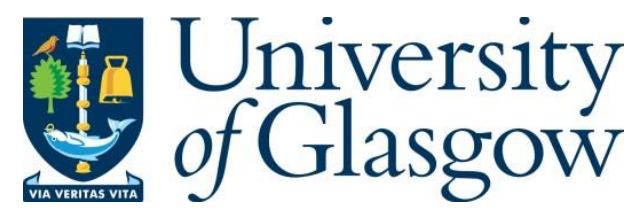

Vuckovic, A., Pangaro, S. and Putri, F. (2018) Unimanual vs bimanual motor imagery classifiers for assistive and rehabilitative brain computer interfaces. IEEE Transactions on Neural Systems and Rehabilitation Engineering,

(doi:10.1109/TNSRE.2018.2877620).

There may be differences between this version and the published version. You are advised to consult the publisher's version if you wish to cite from it.

$\underline{\text { http://eprints.gla.ac.uk/172128/ }}$

Deposited on: 29 October 2018

Enlighten - Research publications by members of the University of Glasgow http://eprints.gla.ac.uk 


\title{
Unimanual vs Bimanual Motor Imagery Classifiers for Assistive and Rehabilitative Brain Computer Interfaces
}

\author{
Vuckovic Aleksandra, Pangaro Sara and Putri Finda
}

\begin{abstract}
Bimanual movements are an integral part of everyday activities and are often included in rehabilitation therapies. Yet electroencephalography (EEG) based assistive and rehabilitative brain computer interface (BCI) systems typically rely on motor imagination (MI) of one limb at the time. In this study we present a classifier which discriminates between uni-and bimanual MI. Ten able bodied participants took part in cue based motor execution (ME) and MI tasks of the left (L), right (R) and both (B) hands. A 32 channel EEG was recorded. Three linear discriminant analysis classifiers, based on MI of L-B, B-R and B-$\mathrm{L}$ hands were created, with features based on wide band Common Spatial Patterns (CSP) 8-30 Hz, and band specifics Common Spatial Patterns (CSPb). Event related desynchronization (ERD) was significantly stronger during bimanual compared to unimanual ME on both hemispheres. Bimanual MI resulted in bilateral parietally shifted ERD of similar intensity to unimanual MI. The average classification accuracy for CSP and CSPb was comparable for $L-R$ task $(\mathbf{7 3} \pm 9 \%$ and $75 \pm 10 \%$ respectively) and for L-B task $(\mathbf{7 3} \pm 11 \%$ and $\mathbf{7 0} \pm 9 \%$ respectively). However, for $R$ $B$ task $(67 \pm 3 \%$ and $72 \pm 6 \%$ respectively) it was significantly higher for $\operatorname{CSPb}(p=0.0351)$. Six participants whose $L-R$ classification accuracy exceeded $70 \%$ were included in an on-line task a week later, using the unmodified offline CSPb classifier, achieving $69 \pm 3 \%$ and $66 \pm 3 \%$ accuracy for the L-R and R-B tasks respectively. Combined uni and bimanual BCI could be used for restoration of motor function of highly disabled patents and for motor rehabilitation of patients with motor deficits.
\end{abstract}

Index Terms-Brain computer interface, electroencephalography, motor imagination, bimanual, common spatial patterns, linear discriminant analysis.

\section{INTRODUCTION}

B RAIN computer interface (BCI) based on motor imagination (MI) has wide range of applications, spanning from spellers to assistive devices [1-7]. Yet MI BCI has most often been used as a part of rehabilitative and assistive systems to repair or restore motor functions. This is due to its reliance on the activity of the sensory-motor cortex $[4,6]$. For these applications, MI-BCI is typically combined with virtual reality, functional electrical stimulation or robots. Examples of these applications are MI BCI for rehabilitation of the upper

\footnotetext{
"This work was supported in part by the Indonesian Government $\mathrm{PhD}$ scholarship."

A Vuckovic is with the Biomedical Engineering, School of Engineering, University of Glasgow, G12 8QQ, UK (email: Aleksandra.vuckovic@glasgow.ac.uk).
}

extremities in stroke patients and patients with incomplete spinal cord injury [4-6] and assistive devices for people with complete high level spinal cord injury (tetraplegia) for restoration of motor functions [2,3].

Although many activities of daily living require use of both hands [8], most MI BCI rehabilitation systems have been designed to assist single limb movements [1-7]. Applications range from the detection of MI of different limbs to detection of direction, speed and target location of a single limb [9-12].

There are however multiple evidences that bimanual training facilitates rehabilitation after stroke [13]. It is believed that during bimanual training, there is no interhemispheric inhibition of the non-targeted hand which occurs during unimanual training. This allows ipsilateral (undamaged) hemisphere and descending pathways to contribute more to the movement of the hemiplegic limb [14]. A BCI which could classify between the unimanual movements of a dominant hand vs bimanual movements, could be used, combined with robotics or functional electrical stimulation (FES) in rehabilitation after stroke or spinal cord injury.

Current trends in assistive MI BCI (e.g. "MoreGrasp" [15]) is to combine $\mathrm{BCI}$ with multi electrode arrays which may enable different grasp patterns [16], but are limited to unimanual control. People with complete tetraplegia, who have bilateral upper limb paralysis, would benefit from assistive devices that would allow a selection between uni- and bimanual tasks, enabling wider range of motor tasks, increasing patients' independence. Combined unilateral and bilateral BCI-FES assisted movements could also be used in rehabilitation of movement in patients with incomplete subacute tetraplegia, because they require therapy of both hands [5].

An asynchronous, EEG based BCI that can detect right, left and bimanual movement has been used to control drone $[17,18]$ or a robotic arm [19] but has been tested on able-bodied people. Participants were trained to control time-varying spectral component amplitude from selected electrodes. The algorithm relied on spatially distinctive event related desynchronsiation (ERD). That however required extensive training of up to 20 hours over several months [17]. In its simplest version, the algorithm required a control of the sensory -motor rhythm recorded at $\mathrm{C} 3$ and $\mathrm{C} 4$ electrodes only. $\mathrm{BCI}$ users produced

S Pangaro was a visiting researcher at the University of Glasgow, while she was with the Universita Campus Bio-medico di Roma, Italy (email: sara.pangaro@hotmail.it). She is now with Johnson\&Johnson, Italy.

F Putri is with the Biomedical Engineering, School of Engineering, University of Glasgow, G12 8QQ, UK (email: f.putri.1@ research.gla.ac.uk). 
ERD at $\mathrm{C} 3$ for $\mathrm{MI}$ of the right hand, over $\mathrm{C} 4$ for the left hand and over both C3 and C4 for bilateral movements [18]. This algorithm could not be used for rehabilitation of stroke patients or patients with spinal cord injury (SCI) from two reasons: first, for patients, initial training time should be minimized in order to start a therapy as soon as possible after injury. Second, in stroke patients clear lateralisation of ERD is absent and the intensity of ERD during MI depends on the site of the lesion [20]. In subacute SCI patients lateralization of ERD may also be absent as these patients have bilateral ERD for unimanual movements [5]. In both patient groups it takes multiple therapy sessions to restore lateralized ERD [5]. In case of assistive BCI for chronic SCI patients, which also combines BCI with FES or robots, the lack of lateralization can be even more of an issue due to a disuse based cortical reorganization [21]. Thus, for these purposes, a classification algorithm has to adapt to a patient specific spatial ERD distribution.

The main problem with detecting the cortical activity during execution or imagination of bimanual movements is that they do not represent a simple superposition of the activity of the sensory-motor cortex during unimanual movements of both hands. It is believed that the main marker of bimanual movements is the strength of connectivity between premotor and parietal areas within and between hemispheres rather than the intensity of activation [22]. Furthermore, cortical areas activated during bimanual movements overlap with the cortical areas activated during unimanual movements, but have stronger activity over the parietal cortex [22,23]. EEG/MEG studies of oscillatory brain activity differentiate between bimanual movements which simultaneously activate homologues muscles, i.e. same flexor muscles of both left and right hand ("in-phase" movement) from those which alternately activate homologues muscles, i.e. right hand flexor and left hand extensor muscles ("out-of-phase" movement) [24]. These studies suggest that in- phase movements present "a default mode" resulting in lower interhemispheric beta band coherence than anti phase and unimanual movements. Increased complexity of movements was associated with increased coherence and increased ERD over a wide band spectral power (alpha, beta and gamma). Biamanual activities of daily living frequently include "in-phase" movements, like for example both hand flexing to grasp a large object. Less pronounced ERD during "in-phase" movements indicate that they might be harder to detect and classify in an BC paradigm than "our-ofphase" movements.

Intensity of ERD depends not only on the complexity of movement but also on hand dominance. Left and bimanual movements showed similar ME ERD in the alpha band, which was larger than ERD during movement of the dominant right hand [25]. While differences between bimanual and unimanual ME have been previously studied, differences in MI ERD between uni- and bimanual movement were however much less investigated.

In this study we compare scalp distribution of ERD between uni- and bimanual MI and ME. We then created a uni- vs bimanual MI classifier and compared it with a left vs right hand MI classifier in both off line and on line paradigms.

\section{METHODS}

Ten adult, right handed able bodied volunteers took part in the study $(29.6 \pm 5.7,5 \mathrm{M}, 5 \mathrm{~F})$ organized over two days. On the first day they participated in a cue-based off-line ME and MI task with one or both hands. Those whose offline left vs right MI classification accuracy exceeded $70 \%$, took part in a subsequent on-line BCI task about a week later. The experiment was approved but the University College Ethical Committee. All participants signed the consent form prior to taking part in the study.

\section{A. Questionnaires}

At the beginning of the off-line session participants filled out the Edinburgh Handedness Inventory questionnaire [26]. Since bimanual movements are predominantly controlled by the dominant hemisphere [22] the level of the hand dominance may have an effect on the classification accuracy. The questionnaire asked participants to select their limb preference for 10 activities of daily living. Handedness was expressed as a laterality index LI

$$
L I=100 \cdot \frac{\sum_{R}-\sum_{L}}{\sum_{R}+\sum_{L}}
$$

where $\Sigma_{\mathrm{R}}$ and $\Sigma_{\mathrm{L}}$ are scores for the right and the left upper limbs. LI can range from -100 to 100 . Negative scores indicate left handed person, positive scores right handed while ambidexterous persons have scores close to 0 on both sides. A larger absolute value indicate stronger hand dominance.

Imagination ability has also been related to the classification accuracy between the left and right hand [27]. Participants' MI ability was tested using Vividness of Movement Imagery Questionnaire (VMIQ) [28]. VMIQ tests three types of MI: external visual imagery (third person imagery), internal visual imagery (first person imagery) and kinaesthetic imagery (first person imagery of sensation in muscles). For the purpose of this study we were primarily interested in kinaesthetic imagery. Kinaesthetic imagery test had 12 questions which were rated in a range from 1 (no sensation) to 5 (sensation as intense as executing the action).

Following the off-line session participants were asked to rate the difficulty of three MI tasks on a Likert type scale ranging from 1 (very difficult) to 5 (very easy). More difficult tasks might recruit more neuronal resources and result in stronger ERD [29], thus potentially being easier to classify. The reason for including these questions was that VMIQ tests the imagination of complex body movements only.

\section{B. Off-line Experimental Sessions}

Participants sit comfortably $1 \mathrm{~m}$ from a computer screen. At $\mathrm{t}=0 \mathrm{~s}$ a warning sign appeared on the computer screen and stayed there until $t=4 \mathrm{~s}$. At $\mathrm{t}=1 \mathrm{~s}$ a warning sign was overlaid by an execution cue ( $\rightarrow$ for the right hand, $\leftarrow$ for the left hand and $\uparrow$ for both hands) which remained on the screen for $1.25 \mathrm{~s}$ (Fig. 1). Depending on a cue, participants executed or imagined to slowly wave with one or with both hands in-phase from $t=1 \mathrm{~s}$ till $\mathrm{t}=4 \mathrm{~s}$. Fifty ME trials of each type (legt, right, both) were followed by one hundred trials of each of three types of MI, divided into 10 shorter sub-sessions. In each sub session, $10 \mathrm{MI}$ of all three types (30 in total) were presented in a semi-random order. 


\section{Off-line EEG Recording and Pre-Processing}

Participants' EEG was measured with 31 electrodes placed according to $10 / 10$ system [30] using g.USBamp device (Guger technologies, Austria). Electrodes covered the central region of the sensory-motor cortex, parietal and frontal cortex, as shown in Fig 2 and 3. One electrode was placed at the lateral cantus of the orbicularis oculi of the right eye to record the electrooculogram (EOG). EEG was recorded with respect to the linked-ear reference with the sampling frequency of 256 samples/s. Impedance was kept under $5 \mathrm{k} \Omega$. A ground electrode was placed at electrode location Fpz. EEG signal was filtered on-line between 0.5 and $60 \mathrm{~Hz}$ and notch filtered at $50 \mathrm{~Hz}$ using an IIR digital Butterworth filter built into a modular amplifier.

For the purpose of calculating Independent Components (IC) [31] and minimizing remaining line nose EEG was further filtered between 2 and $45 \mathrm{~Hz}$. IC related to eye movement artifact, muscle activity or external noise were identified based

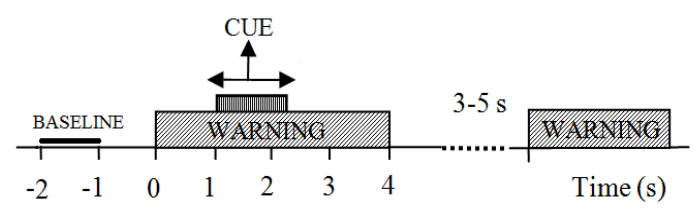

Fig. 1. Experimental paradigm: a warning cue appears at $t=0$ s and stays on a screen until $\mathrm{t}=4$. An execution cue (an arrow) appears at $\mathrm{t}=1 \mathrm{~s}$ and stays on the screen for $1.25 \mathrm{~s}$. Arrows pointing to the left, right and upwards appear in a semi-random order and correspond to MI or ME of the left, right or both hands respectively. ME and $\mathrm{MI}$ were performed in separate sub-sessions. Time between two trials was semi random, between 3 and $5 \mathrm{~s}$. Baseline was calculated from a period $\mathrm{t}=-2 \mathrm{~s}$ to $-1 \mathrm{~s}$ before each warning cue.

on the characteristic spatial location and distribution, frequency content and morphology (blinking and eye movements). Datasets of each participant, containing MI of all three types, were decomposed into independent components (IC) [31] using Infomax algorithm implemented in EEGlab [32] under Matlab (Mathworks). Components were visually inspected and those corresponding to noise (line noise, EOG, EMG and ECG) were removed and signal was back projected into EEG domain. Common average reference was computed for all channels.

\section{Features Extraction}

Event-related spectral perturbation (ERSP) [33] was computed on EEG of executed and imagined movement to compare event-related synchronization/desynchronization (ERS/ERD) [34] between different tasks over a range of frequencies, and to define frequency bands with strongest ERD. The ERSP analysis was performed using Morlet wavelet transform in the frequency band 3-45 Hz. The Hanning-tapered window was applied and the number of cycles of the wavelet was set to 3 . These wavelet settings enabled low frequencies beginning from $3 \mathrm{~Hz}$ to be processed in a one second window. The ERSP was calculated as power changes in decibels with a reference to a baseline period (from $-2 \mathrm{~s}$ to $-1 \mathrm{~s}$ before the warning sign, Fig.1). The ERSD was averaged over trials within the same group, separated according to the imagination condition. A scalp map based on averaged ERS/ERD over a specific time window and over a specific frequency band was created for a group analysis. A time window for plotting ERSP was chosen to match the time window for best classification accuracy, explained further in the text.

The Common spatial patterns (CSP) method [35] was applied to design spatial filters applied on pairs of classes (left-vs right, left vs bi-manual, right vs bi-manual). The CSP method projects multi-channel EEG data into a low-dimensional spatial subspace in such a way that the variances of the filtered time series are optimal for discrimination. CSP filtering minimises the variance of one of the classes while maximising that of the other. The CSP method was applied either to EEG filtered in 8$30 \mathrm{~Hz}$ band (called CSP further in the text) or on pre-defined bands (CSPb) based on observation of ERSD maps [36].

While CSP uses a transformation matrix (spatial filter) $W$ to decompose EEG signal $X^{k}$ of $k$ trials, into time series $Z^{k}$

$$
Z^{k}=W \cdot X^{k}
$$

the $\mathrm{CSPb}$ extends the transformation matrix $W^{(0)}$ with one delayed coordinate $W^{(\tau)}$, where $\tau$ is a delay time.

$$
Z^{k}=W^{(0)} \cdot X^{k}+W^{(\tau)} \cdot \delta^{\tau} \cdot X^{k}
$$

A delayed vector $\delta^{T} X^{k}$ simply appends as an additional channel to $X^{k}$ so that transformation can be presented similar to (2) but transformation matrix $W$ now consists of a spatial and a FIR filter which are interchangeable (one can first apply spatial or frequency filter). This is relevant for on-line classification where FIR filter is applied after spatial filter to a subset of selected projected channels.

In this study, $\mathrm{CSPb}$ were calculated for each participants based on their individual ERD, selecting one or two bands out of preselected frequency bands: $8-12 \mathrm{~Hz}, 16-24 \mathrm{~Hz}, 16-30 \mathrm{~Hz}$ and 30-35 Hz.

The CSP and CSPb filters were computed using data segments that fell within $t=1.5-4$ s following the warning cue. Data in these segments should provide the most significant discriminant features for classifying between classes. Time delay $\tau$ for $\mathrm{CSPb}$ were calculated for $0.125 \mathrm{~s}$ long sub-segments.

\section{E. Features Classification}

Classification was based on Linear Discriminant Analysis (LDA) [37]. This is a technique used to project data onto a low dimensional space to enable the separation of the data into classes. The classes were separated using a hyperplane that maximizes linear separability of the data. To achieve this, LDA maximises a ratio between inter class variance and intra class variance. Classification was performed for each 0.125 s long sub-segment to find a segment with the highest Cohen's kappa [38]. That segment from all but one trail was used to train a classifier. Classifier was then tested on the full length of each trial from $t=1.5$ to $t=4 \mathrm{~s}$ (leave-one-out procedure) for both CSP and CSPb. Classification was implemented in rtsBCI v. 020, Biosig [39], under Matlab (Mathworks, USA). Classification performance was expressed in terms of classification accuracy, sensitivity and specificity.

$$
\text { Accuracy }=\frac{T P+T N}{T P+F P+T N+F N}
$$




$$
\begin{aligned}
& \text { Sensitivity }=\frac{T P}{T P+F N} \\
& \text { Specificity }=\frac{T N}{T N+F P}
\end{aligned}
$$

where TP is true positive, $\mathrm{FP}$ is false positive, TN is true negative and $\mathrm{FN}$ is false negative rate.

Receiver operating characteristic (ROC) was obtained for both classes (specificity vs sensitivity for a range of thresholds) and Area Under Curve (AUC) was calculated. The AUC value corresponding to the time window with highest kappa was reported [40]. All calculations were implemented in Biosig under Matlab.

\section{F On-line Experiment}

The experimental procedure was similar to the off-line procedure, but a bar proportional to the on-line accuracy overlaid the execution cues on a computer screen. The bar was extended to the left or to the right for classification between left and right MI task and to the right and upwards for the classification between the right hand and bimanual MI task.

The unmodified classifier from the off-line session was used. Two classifiers were used: left vs right hand and right vs bimanual. . On-line classification between left hand and both hands MI was not performed to avoid fatigue due to increased concentration, which could result in a reduced performance. Right vs both hand classification was selected as being more relevant for performing activities of daily living of right handed people. For each classifier participants were asked to perform 40 trials per condition i.e. each class (80 trials in total). The order of tested classifiers varied between participants to counterbalance fatigue.

\section{G. Statistical Analysis}

To produce ERSP maps, a statistical non-parametric, bootstrapping analysis, with a significance level $p=0.05$ was used in order to assess the differences between the groups and conditions. A correction for multiple comparisons was performed using the false discovery rate method [41]. Classification results and results of questionnaires were tested for normality using Kolmogorov-Smirnov test. Parametric analysis (paired student t-test) was applied to compare the equality of means of normally distributed data (Acc results in tables 1 and 2) while non parametric rank sum test was applied for data which did not have a normal distribution such as results extracted from questionnaires. A Pearson correlation was calculated between classification accuracy and results of questionnaires. In all tests a significance threshold was set to $\mathrm{p}=0.05$. All analysis was implemented in Matlab (Mathworks).

\section{RESULTS}

Quantitative EEG analysis is presented first followed by the off line and on line classification results.

\section{A. Event Related Spectral Perturbation}

Fig. 2 shows ERSP scalp maps in the alpha $(8-12 \mathrm{~Hz})$ and beta $(13-30 \mathrm{~Hz})$ band during real and imagined movements of
TABLE I

\begin{tabular}{|c|c|c|c|c|c|c|c|}
\hline$\overline{P \mathrm{P}}$ & Ml & $\begin{array}{l}\text { Acc } \\
(\%)\end{array}$ & $\begin{array}{l}\text { Sens } \\
(\%)\end{array}$ & $\begin{array}{l}\text { Spec } \\
(\%)\end{array}$ & $\begin{array}{c}\text { AUC } \\
(\%)\end{array}$ & "KI & 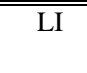 \\
\hline $\bar{~} 1$ & $\begin{array}{l}\text { LR } \\
\text { BR } \\
\text { BL }\end{array}$ & $\begin{array}{l}69 \\
66 \\
68\end{array}$ & $\begin{array}{l}70 \\
65 \\
68\end{array}$ & $\begin{array}{l}67 \\
66 \\
69\end{array}$ & $\begin{array}{l}70 \\
65 \\
69\end{array}$ & 2.4 & 80 \\
\hline 2 & $\begin{array}{l}\text { LR } \\
\text { BR } \\
\text { BL }\end{array}$ & $\begin{array}{l}68 \\
65 \\
67\end{array}$ & $\begin{array}{l}69 \\
65 \\
67\end{array}$ & $\begin{array}{l}67 \\
64 \\
66\end{array}$ & $\begin{array}{l}70 \\
67 \\
66\end{array}$ & 3.0 & 58 \\
\hline 3 & $\begin{array}{l}\text { LR } \\
\text { BR } \\
\text { BL }\end{array}$ & $\begin{array}{l}66 \\
64 \\
64\end{array}$ & $\begin{array}{l}66 \\
64 \\
64\end{array}$ & $\begin{array}{l}66 \\
64 \\
64\end{array}$ & $\begin{array}{l}62 \\
64 \\
66\end{array}$ & 1.2 & 93 \\
\hline 4 & $\begin{array}{l}\text { LR } \\
\text { BR } \\
\text { BL }\end{array}$ & $\begin{array}{l}86 \\
68 \\
89\end{array}$ & $\begin{array}{c}100 \\
68 \\
100\end{array}$ & $\begin{array}{l}81 \\
53 \\
82\end{array}$ & $\begin{array}{l}87 \\
66 \\
91\end{array}$ & 1.4 & 38 \\
\hline 5 & $\begin{array}{l}\text { LR } \\
\text { BR } \\
\text { BL }\end{array}$ & $\begin{array}{l}63 \\
65 \\
71\end{array}$ & $\begin{array}{l}62 \\
65 \\
73\end{array}$ & $\begin{array}{l}65 \\
65 \\
70\end{array}$ & $\begin{array}{l}65 \\
65 \\
69\end{array}$ & 2.4 & 73 \\
\hline 6 & $\begin{array}{l}\text { LR } \\
\text { BR } \\
\text { BL }\end{array}$ & $\begin{array}{l}90 \\
68 \\
69\end{array}$ & $\begin{array}{c}84 \\
100 \\
69\end{array}$ & $\begin{array}{c}100 \\
83 \\
70\end{array}$ & $\begin{array}{l}89 \\
70 \\
70\end{array}$ & 3.1 & 67 \\
\hline 7 & $\begin{array}{l}\text { LR } \\
\text { BR } \\
\text { BL }\end{array}$ & $\begin{array}{l}68 \\
68 \\
69\end{array}$ & $\begin{array}{l}72 \\
73 \\
76\end{array}$ & $\begin{array}{l}61 \\
64 \\
59\end{array}$ & $\begin{array}{l}65 \\
67 \\
70\end{array}$ & 2.9 & 87 \\
\hline 8 & $\begin{array}{l}\text { LR } \\
\text { BR } \\
\text { BL }\end{array}$ & $\begin{array}{l}73 \\
65 \\
68\end{array}$ & $\begin{array}{l}73 \\
64 \\
67\end{array}$ & $\begin{array}{l}74 \\
66 \\
68\end{array}$ & $\begin{array}{l}74 \\
66 \\
66\end{array}$ & 2.4 & 86 \\
\hline 9 & $\begin{array}{l}\text { LR } \\
\text { BR } \\
\text { BL }\end{array}$ & $\begin{array}{l}75 \\
74 \\
72\end{array}$ & $\begin{array}{l}78 \\
82 \\
78\end{array}$ & $\begin{array}{l}73 \\
64 \\
61\end{array}$ & $\begin{array}{l}73 \\
70 \\
70\end{array}$ & 2.3 & 78 \\
\hline 10 & $\begin{array}{l}\text { LR } \\
\text { BR } \\
\text { BL }\end{array}$ & $\begin{array}{l}77 \\
71 \\
73\end{array}$ & $\begin{array}{l}65 \\
61 \\
67\end{array}$ & $\begin{array}{l}62 \\
65 \\
68\end{array}$ & $\begin{array}{l}79 \\
73 \\
73\end{array}$ & 2.6 & 88 \\
\hline Av & $\begin{array}{l}\text { LR } \\
\text { BR } \\
\text { BL }\end{array}$ & $\begin{array}{c}73 \pm 9 \\
67 \pm 3 \\
73 \pm 11\end{array}$ & $\begin{array}{c}74 \pm 11 \\
71 \pm 12 \\
68 \pm 7\end{array}$ & $\begin{array}{c}72 \pm 12 \\
65 \pm 7 \\
68 \pm 7\end{array}$ & $\begin{array}{l}72 \pm 9 \\
67 \pm 3 \\
71 \pm 7\end{array}$ & $2.4 \pm 0.6$ & $75 \pm 17$ \\
\hline
\end{tabular}

RESULTS OF CSP CLASSIFIERS AND OF QUESTIONNAIRES

CSP common spatial patterns; $\mathrm{P}=$ participant; $\mathrm{MI}=$ motor imagery, Acc=accuracy; Sen=sensitivity; Spe=specificity, AUC=area under ROC curve, same for the left and right hand, STD standard deviation. $\mathrm{P}=$ participant, $\mathrm{LR}=$ left vs right; $\mathrm{BR}=$ both vs right, $\mathrm{BL}=$ both vs left, $\mathrm{Av}=$ mean $\pm \mathrm{STD}, \mathrm{KI}=$ kinaesthetic imagery questionnaire, $\mathrm{LI}=$ laterality index.

the left hand, right hand and both hands, averaged over time period $\mathrm{t}=1.5 \mathrm{~s}$ to $2 \mathrm{~s}$ following a warning cue. For real movements in the alpha band there was a statistically significant difference at electrode location $\mathrm{C} 3$ between bimanual and right hand ME task; at C3, C5 and CP5 between the bimanual and left hand ME task; and at C5, CP2, CP4 and P4 between the left and right hand ME task. In the beta band there was a statistically significant difference at $\mathrm{CF} 2$ and $\mathrm{CF} 4$ between bimanual and right hand ME task and at CF4 between bimanual and left hand ME task. In the gamma band statistically significant difference were found only between bimanual and left hand task at CF4 (Fig. 3). In all cases, the bimanual movements had stronger ERD than the unimanual movements.

During imagined movements there was however no statistically significant differences between different types of movements. Looking at lateralization, in the alpha band, MI of left hand and of both hands showed clear ERD lateralization to the right hemisphere. In beta band for all three types of MI, 


\section{MOTOR EXECUTION}
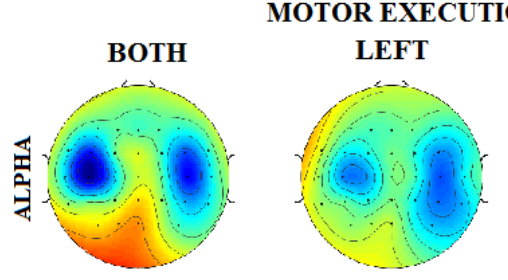

(a)
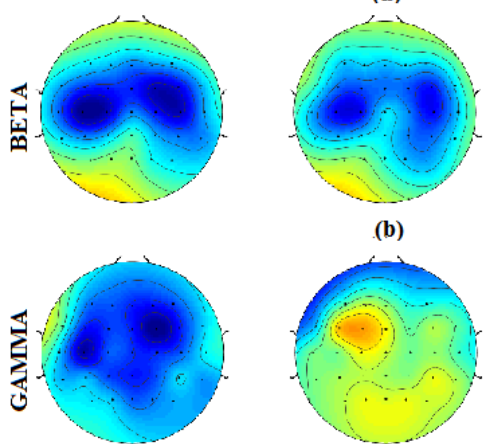

(b)

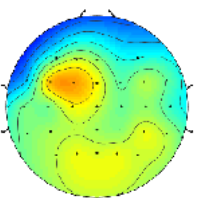

(c)
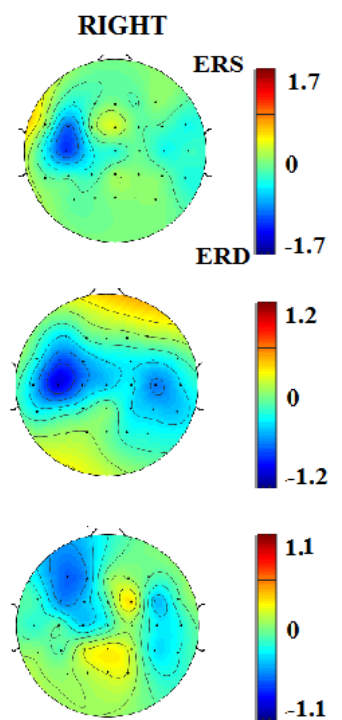
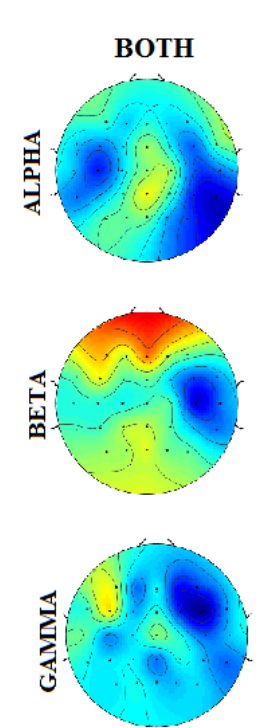

MOTOR IMAGINATION

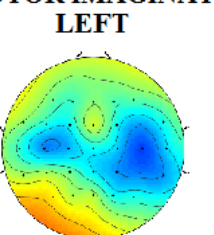

(d)

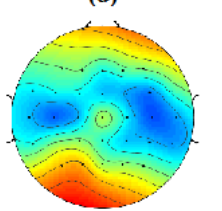

(e)

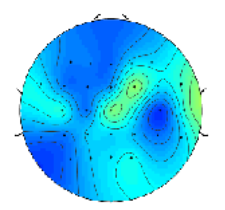

(f)
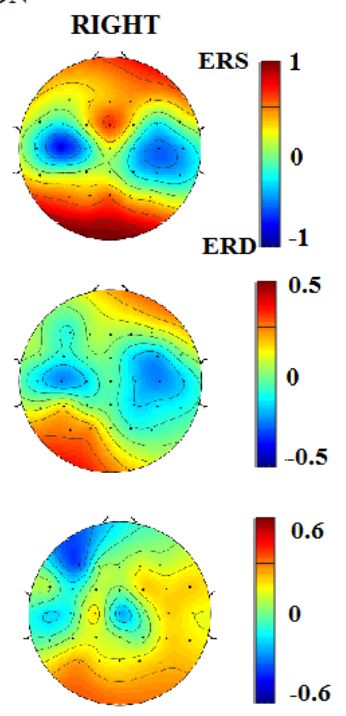

Fig. 2. Spatial maps of group average ERSP (a-c) real movements, (d-f) imagined movements in the alpha, beta and lower gamma (30-40 Hz) band. B: bimanual, $\mathrm{L}$ : left, R: right. Time window from 1.5 to $2 \mathrm{~s}$ post warning cue $(0.5 \mathrm{~s}$ to $1 \mathrm{~s}$ post execution cue).

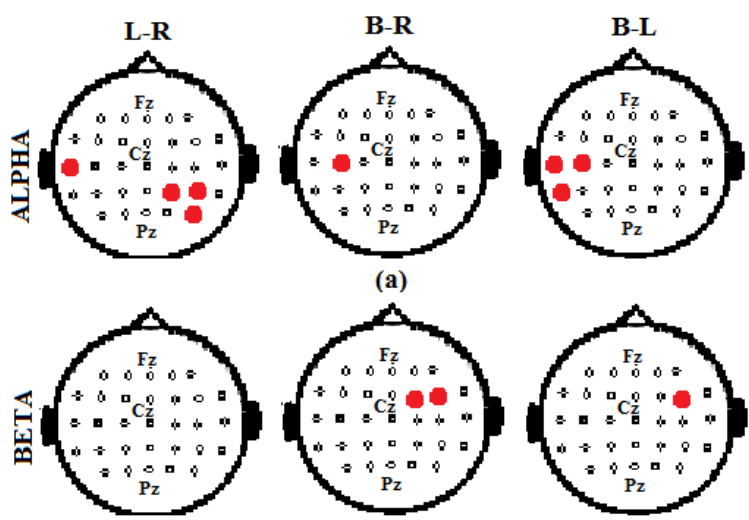

(b)

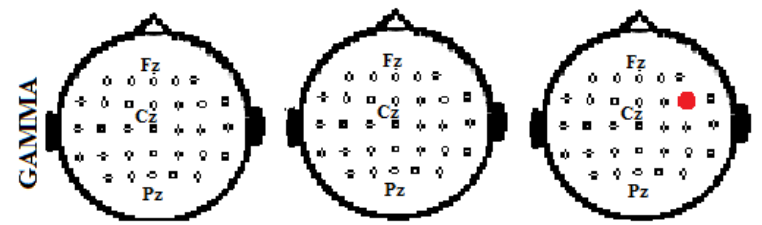

(c)

Fig. 3. Spatial maps showing group level statistically significant differences $(\mathrm{p}=0.05)$ of ERD between different real movements. Electrode locations according to 10-10 system (a) alpha band, (b) beta band, (c) lower gamma band $(30-40 \mathrm{~Hz}), \mathrm{B}$ : bimanual, L: left, R: right. Time window from $0.5 \mathrm{~s}$ to $1 \mathrm{~s}$ post execution cue. Electrode locations (from left to right) top row: F3, F1, Fz, F2, F4; second row: CF5, CF3, CF1, CFz, CF2, CF4, CF6; third (middle) row: $\mathrm{C} 5, \mathrm{C} 3, \mathrm{C} 1, \mathrm{Cz}, \mathrm{C} 2, \mathrm{C} 4, \mathrm{C} 6$; fourth row: CP5, CP3, CP1, CPz, CP2, CP4, CP6, sixth (bottom) row: P3, P1, Pz, P2, P4.

ERD was lateralized to the right (non-dominant hemisphere). Left and both hand MI lateralization was similar to the corresponding ME lateralization. In the gamma band ERD was lateralized to the right for bimanual MI. For MI of the left hand ERD was widespread bilateraly and no clear ERD could be noticed for right hand MI.

In summary, both real and imagined bimanual movements resulted in bilateral desynchronization, more wide spread over the non-dominant hemisphere. For real movements, in all three bands, ERD was stronger during bimanual than during unimanual movements. These results indicate that feature extraction methods based on spatial filters might be adequate to classify between the uni- and bimanual movements. Largest differences between uni and bimanual movements can be noticed over the left (dominant) hemisphere for the alpha band and over the right hemisphere for the beta and gamma bands.

\section{B. Off-line Classification}

Table 1 shows classification performance for CSP classifiers (accuracy, sensitivity and specificity and AUC) for individual participants. It also shows kinesthetic imagery and LI scores for each single participant. The average classification accuracy was for left vs right (L-R) hand $73 \pm 9 \%$, for both vs right hand (BR) $67 \pm 3 \%$ and for both vs left hand (B-L) $71 \pm 9 \%$. There was no statistically significant differences between R-L and B-L as well as between B-R and B-L accuracy but there was a statistically significant difference between the classification accuracy of $\mathrm{L}-\mathrm{R}$ and $\mathrm{B}-\mathrm{R}$ ( $\mathrm{p}=0.0372$, paired $\mathrm{t}$ test). The average kinaesthetic imagery was moderate $2.4 \pm 0.6$ and the average LI was $75 \pm 17$, meaning that participants were on average clearly right handed. The average self-reported difficulty of imagination of hand movements of a single hand was 2.4 \pm 0.7 and $2.4 \pm 0.7$ for the right and for the left hand respectively, while for both hands it was $2.6 \pm 1.0$. There were no statistically significant differences in self-reported difficulty of MI between tasks.

Table 2 shows classification results for CSPb and frequency bands for which best accuracy was achieved. The average classification accuracy was for L-R $75 \pm 10 \%$, for B-R $72 \pm 6 \%$

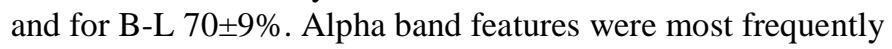
selected, although in 4 participants, single beta band features achieved the best accuracy for either R-B or L-B classification. Lower gamma band $(30-35 \mathrm{~Hz})$ was selected only three times 
TABLE II

RESULTS OF OFF-LINE CSPB CLASSIFIER

\begin{tabular}{|c|c|c|c|c|c|c|}
\hline $\bar{P}$ & Ml & Band (Hz) & $\begin{array}{l}\text { Acc } \\
(\%)\end{array}$ & $\begin{array}{l}\text { Sen } \\
(\%)\end{array}$ & $\begin{array}{c}\text { Spec } \\
(\%)\end{array}$ & $\begin{array}{c}\text { AUC } \\
(\%)\end{array}$ \\
\hline 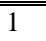 & "LR & 8-12;16-30 & 66 & 65 & 66 & 866 \\
\hline & $\begin{array}{l}\text { BR } \\
\text { BL }\end{array}$ & $\begin{array}{l}8-12 \\
8-12 ; 30-35\end{array}$ & $\begin{array}{l}65 \\
64\end{array}$ & $\begin{array}{l}66 \\
64\end{array}$ & $\begin{array}{l}65 \\
65\end{array}$ & $\begin{array}{l}67 \\
62\end{array}$ \\
\hline 2 & $\begin{array}{l}\text { LR } \\
\text { BR } \\
\text { BL }\end{array}$ & $\begin{array}{l}16-30 \\
8-12 ; 16-30 \\
8-12 ; 30-35\end{array}$ & $\begin{array}{l}65 \\
66 \\
67\end{array}$ & $\begin{array}{l}64 \\
65 \\
68\end{array}$ & $\begin{array}{l}68 \\
66 \\
66\end{array}$ & $\begin{array}{l}68 \\
65 \\
66\end{array}$ \\
\hline 3 & $\begin{array}{l}\text { LR } \\
\text { BR } \\
\text { BL }\end{array}$ & $\begin{array}{l}8-12 ; 16-30 \\
8-12 \\
8-12\end{array}$ & $\begin{array}{l}65 \\
69 \\
63\end{array}$ & $\begin{array}{l}65 \\
69 \\
62\end{array}$ & $\begin{array}{l}66 \\
69 \\
64\end{array}$ & $\begin{array}{l}62 \\
67 \\
64\end{array}$ \\
\hline 4 & $\begin{array}{l}\text { LR } \\
\text { BR } \\
\text { BL }\end{array}$ & $\begin{array}{l}8-12 ; 16-30 \\
8-12 ; 16-30 \\
8-12 ; 16-30\end{array}$ & $\begin{array}{l}90 \\
76 \\
90\end{array}$ & $\begin{array}{l}83 \\
77 \\
89\end{array}$ & $\begin{array}{c}100 \\
74 \\
90\end{array}$ & $\begin{array}{l}89 \\
74 \\
90\end{array}$ \\
\hline 5 & $\begin{array}{l}\text { LR } \\
\text { BR } \\
\text { BL }\end{array}$ & $\begin{array}{l}8-12,16-24 \\
16-24 \\
16-24\end{array}$ & $\begin{array}{l}65 \\
63 \\
60\end{array}$ & $\begin{array}{l}64 \\
61 \\
59\end{array}$ & $\begin{array}{l}65 \\
65 \\
61\end{array}$ & $\begin{array}{l}66 \\
61 \\
61\end{array}$ \\
\hline 6 & $\begin{array}{l}\text { LR } \\
\text { BR } \\
\text { BL }\end{array}$ & $\begin{array}{l}8-12 ; 16-24 \\
8-12 ; 16-30 \\
8-12,16-24\end{array}$ & $\begin{array}{l}90 \\
83 \\
70\end{array}$ & $\begin{array}{l}96 \\
80 \\
70\end{array}$ & $\begin{array}{l}85 \\
86 \\
71\end{array}$ & $\begin{array}{l}85 \\
84 \\
69\end{array}$ \\
\hline 7 & $\begin{array}{l}\text { LR } \\
\text { BR } \\
\text { BL }\end{array}$ & $\begin{array}{l}8-12 ; 16-24 \\
8-12 ; 16-30 \\
8-12,30-35\end{array}$ & $\begin{array}{l}72 \\
75 \\
66\end{array}$ & $\begin{array}{l}62 \\
77 \\
62\end{array}$ & $\begin{array}{l}84 \\
73 \\
69\end{array}$ & $\begin{array}{l}70 \\
75 \\
69\end{array}$ \\
\hline 8 & $\begin{array}{l}\mathrm{LR} \\
\mathrm{BR} \\
\mathrm{BL}\end{array}$ & $\begin{array}{l}8-12 ; 16-30 \\
8-12 \\
16-24\end{array}$ & $\begin{array}{l}82 \\
79 \\
70\end{array}$ & $\begin{array}{l}83 \\
77 \\
73\end{array}$ & $\begin{array}{l}82 \\
81 \\
68\end{array}$ & $\begin{array}{l}82 \\
80 \\
71\end{array}$ \\
\hline 9 & $\begin{array}{l}\text { LR } \\
\text { BR } \\
\text { BL }\end{array}$ & $\begin{array}{l}16-24 \\
16-30 \\
8-12\end{array}$ & $\begin{array}{l}79 \\
76 \\
76\end{array}$ & $\begin{array}{l}79 \\
67 \\
78\end{array}$ & $\begin{array}{l}80 \\
83 \\
77\end{array}$ & $\begin{array}{l}80 \\
81 \\
76\end{array}$ \\
\hline 10 & $\begin{array}{l}\text { LR } \\
\text { BR } \\
\text { BL }\end{array}$ & $\begin{array}{l}16-30 \\
8-12 ; 16-30 \\
16-30\end{array}$ & $\begin{array}{l}77 \\
70 \\
73\end{array}$ & $\begin{array}{l}79 \\
72 \\
74\end{array}$ & $\begin{array}{l}76 \\
69 \\
73\end{array}$ & $\begin{array}{l}76 \\
69 \\
71\end{array}$ \\
\hline Av & $\begin{array}{l}\text { LR } \\
\text { BR } \\
\text { BL }\end{array}$ & & $\begin{array}{c}75 \pm 10 \\
72 \pm 6 \\
70 \pm 9\end{array}$ & $\begin{array}{c}74 \pm 12 \\
71 \pm 6 \\
70 \pm 9\end{array}$ & $\begin{array}{c}77 \pm 11 \\
73 \pm 8 \\
70 \pm 8\end{array}$ & $\begin{array}{l}71 \pm 9 \\
72 \pm 8 \\
70 \pm 8\end{array}$ \\
\hline
\end{tabular}

$\mathrm{CSPb}$ Band specific common spatial patterns; $\mathrm{P}=$ participant; $\mathrm{MI}=$ motor imagery, Acc=accuracy; Sen=sensitivity; Spe=specificity, AUC= area under ROC curve (same for left and right hand), STD standard deviation $\mathrm{P}=$ participant, $\mathrm{LR}=$ left vs right; $\mathrm{BR}=$ both vs right, $\mathrm{BL}=$ both vs left, $\mathrm{Av}=$ mean \pm STD

and in all cases it was for L-B classification. For CSPb, there was no statistically significant difference in classification accuracy between any of the tasks. There was also no significant difference between CSP and CSPb accuracy for neither L-R nor for B-L task. However for B-R task, classifier based on $\mathrm{CSPb}$ feature had significantly higher classification accuracy ( $\mathrm{p}=0.0351$, paired $\mathrm{t}$ test).

We also investigated a relation between the kinesthetic imagery and classification accuracy (CSPb and CSP features) and a relation between $\mathrm{LI}$ and the classification accuracy. There was no significant correlation between kinesthetic imagery scores and classification accuracy for neither CSP nor CSPb based classifier. Likewise there was no significant correlation between the LI and classification accuracy of any classifier.

Figure 4 shows first two CSP for a representative volunteer (P4) used for classification between two conditions. For L-R classification CSP corresponded to areas over the sensory-
TABLE III

CLASSIFICATION ACCURACY OF ON-LINE CSPB CLASSIFIER [\%]

\begin{tabular}{|c|c|c|c|c|c|c|c|c|}
\hline & & P4 & P6 & P7 & P8 & P9 & P10 & $\begin{array}{l}\text { Mean } \pm \text { STD } \\
{[95 \% \mathrm{CI}]}\end{array}$ \\
\hline $\mathrm{L}$ & Acc & 68 & 64 & 68 & 70 & 73 & 73 & $69 \pm 3\left[\begin{array}{lll}68.7 & 69.3\end{array}\right]$ \\
\hline \multirow[t]{2}{*}{$\mathrm{R}$} & Sen & 65 & 66 & 67 & 68 & 84 & 80 & $72 \pm 8\left[\begin{array}{lll}71.3 & 72.7\end{array}\right]$ \\
\hline & Spe & 73 & 62 & 68 & 63 & 70 & 68 & $67 \pm 4\left[\begin{array}{lll}66.6 & 67.4\end{array}\right]$ \\
\hline B & Acc & 68 & 63 & 62 & 65 & 69 & 66 & $66 \pm 3\left[\begin{array}{ll}65.7 & 66.3\end{array}\right]$ \\
\hline \multirow[t]{2}{*}{$\mathrm{R}$} & Sen & 76 & 61 & 61 & 63 & 71 & 64 & $66 \pm 6[65.466 .5]$ \\
\hline & Spe & 69 & 65 & 70 & 72 & 67 & 70 & $69 \pm 3\left[\begin{array}{ll}68.7 & 69.3\end{array}\right]$ \\
\hline
\end{tabular}

$\mathrm{CSPb}$ Band specific common spatial patterns; Acc=accuracy; Sen=sensitivity; Spe=specificity, STD standard deviation, CI confidence interval. $\mathrm{P}=$ participant, $\mathrm{LR}=$ left vs right; $\mathrm{BR}=$ both vs right

motor cortex. For R-B CSP had a parieto-occipital and left cental location while for L-B they had mid-central and parietooccipital location. For the L-R and R-B classifications, spatial filters over the left hemisphere (covering sensory-motor cortex of the right hand) looked almost identical. Similarly, occipitoparietal spatial filters, being representative for MI of both hands, look almost identical for R-B and L-B classifiers. The spatial filters are the same for CSP and CSPb because the CSP algorithm first calculates spatial filters and then filters CSP time series in different frequency bands.

\section{On-line Classification}

For on-line experiments, $\mathrm{CSPb}$ classifiers were used. Table 3 shows on-line classification accuracy for L-R MI $(69 \pm 3 \%)$ and for B-R MI $(66 \pm 3 \%)$. The average classification accuracy dropped equally, for $6 \%$, for both classifiers as compared to the off-line classifiers. Classification between uni- vs bimanual task had lower accuracy than classification between L-R tasks but is was higher than the chance level which for this number of trials $(\mathrm{N}=80)$ is $60 \%$ [42]. Based on the observation of the accuracy as a function of time in off-line experiment, a period from $1.5 \mathrm{~s}$ to $2 \mathrm{~s}$ following the warning cue was used for classification, as it showed best classification accuracy in all participants.

\section{DISCUSSION}

Activities of daily living combine uni- and bimanual tasks. Yet assistive and rehabilitative BCI typically rely on using one hand
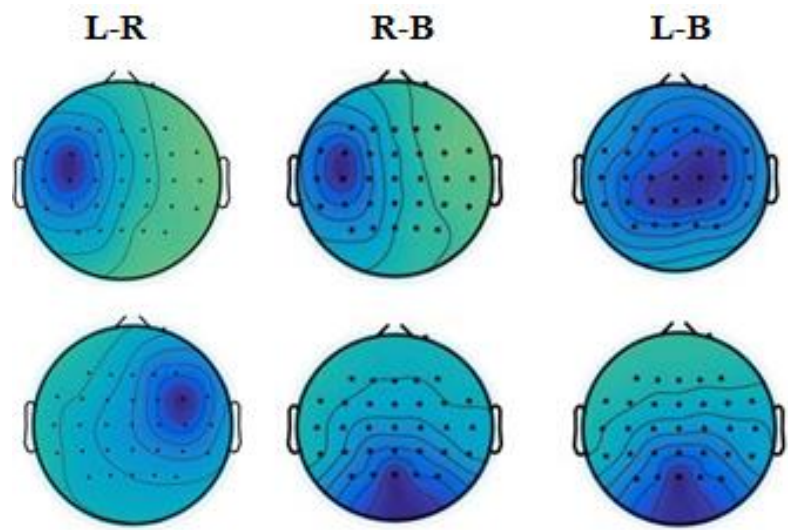

Fig. 4. Spatial distribution of first two CSP for classification between the left and right MI (L-R left columns), right and both hands (R-B middle column) and left and both hands (L-B right column). 
at the time. Creating a BCI classifier between uni- and bimanual MI is considered a difficult task, based on the assumption that a bimanual task does not produce neither stronger nor spatially distinctive cortical activation as compared to the unimanual tasks [22,23].

Results of this study showed that (i) on a group level, both ME and MI of bimanual movement had spatially distinctive ERD patterns different for alpha, beta and gamma bands, (ii) utilizing spatial time frequency distribution it was possible to create a uni-vs bimanual classifier which achieved a comparable classification accuracy to the left vs right hand MI classification.

ME of a bimanual task had significantly stronger ERD than unimanual tasks, and that was more pronounced in the alpha than in the beta and gamma band. Compared to ME of the right and of left hand, ME of the bimanual task resulted in stronger ERD over the left hemisphere for the alpha band and over the right hemisphere for the beta band. Motor execution ERD in the alpha and beta bands of both bimanual and left hand tasks had a bilateral distributions, confirming results from [25]. In the gamma band, clear bilateral ERD was noticed during the bimanual task only, while no ERD was visible during the unimanual tasks. These results demonstrate that algorithms relying on spatially distinctive ERD between uni and bimanual tasks, as suggested in [18], could not be used for untrained participants.

The bimanual MI task had parietal ERD more spread over the non-dominant hemisphere but the intensity was not significantly stronger than during unimanual MI tasks. Contrary to the results of our previous study on another group of healthy participants [43] we found no significant difference between ERD during MI of the left and the right hand. That might explain somewhat lower classification accuracy of L-R classifier in this study vs $83 \pm 3 \%$ in [43]. On average $20 \%$ people cannot use MI BCI [44] which might reflect on differences in classification accuracy between healthy volunteers.

For the on-line experiment we selected only participants whose off-line classification accuracy was $70 \%$ or higher based on recommendations from the literature [45]. This was however recommendation for systems for communication and control but it is not known if this level of accuracy is required for assistive and rehabilitative BCI.

We performed classification based on CSP features which utilized spatially distinctive distribution of ERD and showed that classification between right vs both hands could be further improved when band specific CSPb features were created. What is most important, we showed that uni-vs bimanual and L-R MI classifiers may achieve comparable classification accuracies. Relevant results is also that the specificity, selectivity and AUC were of comparable values as classification accuracy, indicating no particular bias towards false positive or false negative.

Although classification accuracies of the on-line classifiers were lower than that of the off-line classifiers, they dropped proportionally for L-R and B-R tasks. That indicates that lower average accuracy of the on-line classifier was not specific to the task, but rather to a general variability of EEG features between sessions. Performing a short additional off line calibration session [46] on the day of the on-line experiment might have improved the classification accuracy. Further optimization of CSP features could be achieved by simultaneously or alternately optimizing temporal FIR and spatial filters [46-48]. Time window from which feedback is provided may also affect classification accuracy; methods such as correlation -based time window selection could be used to automatically detect optimal time window for each person [49].

Contrary to our previous study [27], we found no correlation between motor imagery ability and classification accuracy. One reason might be that in our previous study we used band power features from 3 bipolar montage channels rather than CSP. Thus unlike this study, the previous study reflected a relation between the power of the sensory-motor rhythm over the central cortex and kinaesthetic imagery. We also did not find any correlation between handedness degree and classification accuracy, probably because most participants had high degree of right handedness.

A limitation of this study was that it has been performed on the able bodied people although the main intended users of the proposed classifier are patients with stroke or spinal cord injury. Stroke affects the laterality of ERD thus MI of bimanual movements might be more lateralized towards the unaffected side, which might influence results based on CSP. We believe that due to the inherent lack of laterality of ERD of bimanual task, uni- vs bimanual MI classifiers might be less affected by stroke than left vs right hand classifiers.

In SCI patients, the level of ERD changes over time [50]. Subactue incomplete patients may have stronger, parietally shifted, less lateralized cortical ERD, which becomes more central and lateralized over the course of recovery [5]. On the other hand, in patients with chronic complete SCI who did not recover motor function of their upper limbs, ERD is weaker than in able-bodied, typically resulting in worse BCI performance [51] unless affected by a secondary condition, such as a chronic pain [52]. Thus it is possible that uni- vs bimanual classifiers would have different performances in rehabilitative BCI used by incomplete subacute SCI patients and in assistive BCI used by chronic complete SCI patients.

Many patents unable to perform a motor task can differentiate between motor imagination and motor attempt [53]. A motor attempt is more similar to motor execution thus potentially creating stronger ERD which are easier to classify than MI, resulting in higher performance of uni-vs bimanual classifier.

\section{CONCLUSIONS}

In this paper, we propose a novel MI BCI paradigm. Uni-vs bimanual classifier has comparable performances to left vs right MI BCI classifiers and could be used in all applications in which MI classifiers are used. We suggest that its main application should be for rehabilitative and assistive BCI where it could increase the variety and complexity of motor tasks. 


\section{REFERENCES}

1. K.K. Ang, C. Guan, "EEG-Based Strategies to Detect Motor Imagery for Control and Rehabilitation," IEEE Trans Neural Syst Rehabil Eng. vol. 25, no. 4, pp. 392-401, Apr 2017

2. M. Rohm, M. Schneiders, C. Müller, A. Kreilinger, V. Kaiser, G.R. Müller-Putz , R. Rupp. "Hybrid brain-computer interfaces and hybrid neuroprostheses for restoration of upper limb functions in individuals with high-level spinal cord injury," Artif Intell Med. vol. 59, no. 2, pp. 133-142, Oct 2013

3. C. Enzinger, S. Ropele, F. Fazekas, M. Loitfelder, F. Gorani, T. Seifert, G. Reiter, C. Neuper, G. Pfurtscheller, G. Müller-Putz. "Brain motor system function in a patient with complete spinal cord injury following extensive brain-computer interface training," Exp Brain Res. vol. 190, no. 2, pp. 215-223, Sep 2008

4. Y.Y. Jang, T.H. Kim, B.H. Lee, "Effects of Brain-Computer Interface-controlled Functional Electrical Stimulation Training on Shoulder Subluxation for Patients with Stroke: A Randomized Controlled Trial." Occup Ther Int. vol 23, no. 2, pp. 175-185, Jun 2016

5. B.C. Osuagwu, L. Wallace, M. Fraser, A. Vuckovic. "Rehabilitation of hand in subacute tetraplegic patients based on brain computer interface and functional electrical stimulation: a randomised pilot study.," J Neural Eng. vol. 13, no. 6 pp. 065002, 2016

6. A.Riccio et al. "Interfacing brain with computer to improve communication and rehabilitation after brain damage," Prog Brain Res. vol. 228, no. 1, pp. 357-387 2016.

7. A. Ramos-Murguialday, D. Broetz, M. Rea, L. Läer, O. Yilmaz, F.L. Brasil, G. Liberati, M.R. Curado, E. Garcia-Cossio, A. Vyziotis, W. Cho, M. Agostini,,E. Soares, S. Soekadar, A. Caria, L.G. Cohen, N. Birbaumer. "Brain-machine interface in chronic stroke rehabilitation: a controlled study," Ann Neurol. Vol. 74, no 1. pp.100-8, Nov 2013

8. S.P. Swinnen. "Intermanual coordination: from behavioural principles to neural-network interactions," Nat Rev Neurosci. Vol. 3, no.5, pp. 348-59, May 2002

9. A. Vuckovic, F. Sepulveda. "Delta band contribution in cue based single trial classification of real and imaginary wrist movements," Med Biol Eng Comput. vol. 46, no. 6, pp. 529-539, Jun 2008.

10. Y. Gu, K. Dremstrup K, D. Farina, "Single-trial discrimination of type and speed of wrist movements from EEG recordings," Clin Neurophysiol. vol 120, no. 8, pp. 1598-1600, Aug 2009.

11. A.Schwarz, P. Ofner, J. Pereira, A.I. Sburlea, G.R. Müller-Putz . "Decoding natural reach-and-grasp actions from human EEG," $J$ Neural Eng. vol 15, no 1, pp. 016005, Feb 2018

12. F. Shiman, E. López-Larraz, A. Sarasola-Sanz, N. Irastorza-Landa, M. Spüler , N. Birbaumer, A. Ramos-Murguialday. "Classification of different reaching movements from the same limb using EEG," $J$ Neural Eng. vol. 14, no 3, pp. 046018, Aug. 2017

13. J.H. Cauraugh, N. Lodha, S.K. Naik, J.J. Summers. "Bilateral movement training and stroke motor recovery progress: a structured review and meta-analysis," Hum Mov Sci. vol. 29, no. 5, pp. 853-70, Oct 2010 .

14. J.H. Cauraugh, J.J. Summers. "Neural plasticity and bilateral movements: A rehabilitation approach for chronic stroke," Prog Neurobiol. Vol 75, no 5, pp. 309-20, Apr 2005.

15. G. R. Müller-Putz, J. Pereira, P. Ofner, A. Schwarz, C. Lopes Dias, R. Kobler, L. Hehenberger, A. I. Sburlea. Towards non-invasive brain-computer interface for hand/arm-control in users with high spinal cord injury, (2018, Jan) Presented at the 6th International Winter Conference on Brain-Computer Interface, South Korea, DOI 10.1109/IWW-BCI.2018.8311498

16. C.T. Freeman, K. Yang, J. Tudor, M. Kutlu. "Feedback control of electrical stimulation electrode arrays," Med Eng Phys. vol. 38, no.11, pp. 1185-1194, Nov 2016.

17. K. LaFleur, K. Cassady, A. Doud, K. Shades, E. Rogin, B. He "Quadcopter control in three-dimensional space using a noninvasive motor imagery-based brain-computer interface," J Neural Eng. vol. 10, no.4, pp:046003, Aug 2013.

18. B.J. Edelman, J. Meng, N. Gulachek, C.C.Cline, B. He. "Exploring Cognitive Flexibility With a Noninvasive BCI Using Simultaneous Steady-State Visual Evoked Potentials and Sensorimotor Rhythms," IEEE Trans Neural Syst Rehabil Eng. vol. 26, no. 5, pp. 936-947, May 2018
19. J. Meng, S. Zhang, A. Bekyo, J. Olsoe, B. Baxter, B. He. "Noninvasive Electroencephalogram Based Control of a Robotic Arm for Reach and Grasp Tasks," Sci Rep. vol. 6, no 12. pp. 38565, Dec 2016.

20. A.M. Ray, E. E. Lopez-Larraz, T.C. Figueiredo, N. Birbaumer, A. Ramos-Murguialday. Movement-related brain oscillations vary with lesion location in severely paralyzed chronic stroke patients,(2017, Jul) Presented at IEEE Eng Med Biol Soc. pp 16641667. DOI 10.1109/EMBC.2017.8037160

21. L. Solstrand Dahlberg, L. Becerra, D. Borsook, C. Linnman "Brain changes after spinal cord injury, a quantitative meta-analysis and review," Neurosci Biobehav Rev. vol 90, no 7. pp. 272-293, Jul 2018

22. A. J. Szameitat, A. McNamara, S. Shen, A. Sterr, "Neural activation and functional connectivity during motor imagery of bimanual everyday actions," PloS One, vol.7 no. 6, pp. e38506, Jun 2012.

23. D.G. Nair, K.L. Purcott, A. Fuchs, F. Steinberg, J.A. Kelso, "Cortical and cerebellar activity of the human brain during imagined and executed unimanual and bimanual action sequences: a functional MRI study," Brain Res Cogn Brain Res. vol. 15, no 3, pp. 250-260, Feb 2003

24. L.M. Rueda-Delgado, E. Solesio-Jofre, D.J. Serrien, D. Mantini, A. Daffertshofer, S.P. Swinnen. "Understanding bimanual coordination across small time scales from an electrophysiological perspective, “Neurosci Biobehav Rev. vol. 47, no 11, pp. 614-35, Nov 2014.

25. M.P. Deiber, R. Caldara, V. Ibañez, C.A. Hauert. "Alpha band power changes in unimanual and bimanual sequential movements, and during motor transitions, "Clin Neurophysiol. Vol. 112, no.8, pp. 1419-35, Aug 2001

26. R.C. Oldfield, "The assessment and analysis of handedness: the Edinburgh inventory," Neuropsychologia. vol. 9, no. 1, Mar 1971

27. A.Vuckovic, B.A. Osuagwu, "Using a motor imagery questionnaire to estimate the performance of a Brain-Computer Interface based on object oriented motor imagery," Clin Neurophysiol. vol. 124, no. 8. pp. 1586-1595, Aug 2013.

28. A. Isaac, D.F. Marks, D.G. Russell, “An instrument for assessing imagery of movement: The Vividness of Movement Imagery Questionnaire (VMIQ)," Journal of Mental Imagery, vol 10, no 4, pp. 23-30, April 1986.

29. J.A. Pineda ."The functional significance of mu rhythms: translating "seeing" and "hearing" into "doing"," Brain Res Brain Res Rev. vol. 50, no. 1, pp. 57-68, Dec 2005

30. "American Clinical Neurophysiology Society, Guideline 5 Guidelines for standard electrode position nomenclature." J Clin Neurophysiol vol 23, pp.107-110, 2006.

31. A.J. Bell, T.J. Sejnowski. "An information-maximization approach to blind separation and blind deconvolution," Neural Comput. vol. 7, no 6, pp. 1129-1159, Nov 1995.

32. A. Delorme, S. Makeig, "EEGLAB: An open source toolbox for analysis of single-trial EEG dynamics including independent component analysis," J Neurosci Methods. vol 134, no 1, pp. 9-21 Mar 2004.

33. S. Makeig, "Auditory event-related dynamics of the EEG spectrum and effects of exposure to tones," Electroencephalogr Clin Neurophysiol. vol 86, no4, pp. 283-293, April 1993.

34. G. Pfurtscheller, F.H. Lopes da Silva. "Event-related EEG/MEG synchronization and desynchronization: basic principles," Clin Neurophysiol. vol. 110, no. 11, pp. 1842-1157, Nov 1999

35. H. Ramoser, J. Mueller-Gerking, and G. Pfurtscheller, "Optimal spatial filtering of single trial EEG during imagined hand movement," IEEE Trans. Rehabil. Eng.,vol. 8, no. 4, pp. 441-446, Apr 2000.

36. S. Lemm, B. Blankertz, G. Curio, K.R. Muller, "Spatio-Spectral Filters for Improving the Classification of Single Trial EEG Biomedical Engineering," IEEE Trans Biomed Eng. vol. 52, No 9, pp. $1541-1548$, Sept. 2005

37. R.O. Duda, P.E. Hart, D.G. Stork. Pattern Classification, 2nd ed. Wiley; 2000.pp. 117-121.

38. M. Billinger, I. Daly, V. Kaiser, B. Allison, G.R.M"uller-Putz, and C. Brunner, Towards practical brain-computer interfaces: bridging the gap from research to real-world applications. Springer Science \& Business Media, 2012, Is it Significant? Guidelines for Reporting BCI Performance, pp. 333-354.

39. C. Vidaurre, T.H. Sander, A. Schlögl."BioSig: the free and open source software library for biomedical signal processing,".Comput 
Intell Neurosci. pp. 935364, Mar 2005, Assessed (03/03/2018) doi: 10.1155/2011/935364 [online]

40. A. Schlögl, J. Kronegg, J.E. Huggins, S.G. Mason. "Evaluation criteria in BCI research", in Towards Brain-Computer Interfacing, G. Dornhege, J. R. Millán, T. Hinterberger, D. McFarland, K.R. Müller Eds Cambridge MA, MIT Press, 2007, Ch 9, pp 327-342 [onine] Available http://pub.ist.ac.at/ schloegl/publications/Schloegl2007_Evaluatio nCriteria.pdf

41. Y. Benjamini, D. Yekutieli, "The control of the false discovery rate in multiple testing under dependency," Ann Statist vol 29 no 4, pp. 1165-1188, Aug 2001.

42. G. R. Müller-Putz, R. Scherer, C. Brunner, R. Leeb, G. Pfurtscheller. "Better than random? A closer look on BCI results," Int. J. Biomagnet. vol 10, no 1, pp. 52-55, 2008.

43. B.C. Osuagwu, M, Zych, A. Vuckovic,"Is Implicit Motor Imagery a Reliable Strategy for a Brain-Computer Interface," IEEE Trans Neural Syst Rehabil Eng. vol. 25, no 12, pp. 2239-2248. Dec 2017.

44. G. Edlinger, B.Z. Allison, C. Guger, "How many people can use a BCI system", in Clin Syst Neurosci, K. Kansaku L. G. Cohen, N. Birbaumer Eds, Japan, Springer, 2015, Ch 3. pp. 33-66

45. A. Kübler, N. Neuman, B. Wilhelm, T. Hinterberger, N. Birbaumer, "Predictability of Brain-Computer Communication," $J$ Psychophysiol. vol 18, pp. 121-129, Sep 2014

46. B. Blankertz, R. Tomioka, S. Lemm, M. Kawanabe, K.R. Muller. "Optimising spatial filters for robust EEG single-trial analysis," IEEE Sig Proc Mag, vol 41, no 1, pp. 41-56, Dec 2008.

47. F. Lotte, C. Guan, "Regularizing common spatial patterns to improve BCI design: unified theory and new algorythms,", IEEE Trans Biomed Eng, vol. 58, no. 2, pp. 355-362, Feb 2011.

48. S.H. Park, S.G. Lee, "Small sample setting and frequency band selection problem solving using subband regularized common spatial patterns," IEEE Sens J., vol. 17, no. 10, pp. 2977-2983, May 2017.

49. J. Feng, E. Yin, J. Jin, R. Saab, I. Daly, X. Wang, D. Hu, A. Cichocki. "Towards correlation-based time window selection method for motor imagery BCIs," Neural Netw.vol. 102, no.6 pp. 87-95, Jun 2018.

50. E. López-Larraz, L. Montesano, A. Gil-Agudo, J. Minguez, A. Oliviero, "Evolution of EEG Motor Rhythms after Spinal Cord Injury: A Longitudinal Study." PLoS One. vol. 10, no. 7, Jul 2015 pp. e0131759.

51. G. Pfurtscheller, P. Linortner, R Winkler, G. Korisek, G. MüllerPutz, "Discrimination of motor imagery-induced EEG patterns in patients with complete spinal cord injury," Comput Intell Neurosci. pp. 104180, Apr 2009

52. A. Vuckovic, M.A. Hasan, B. Osuagwu, M. Fraser, D.B. Allan, B.A. Conway, B. Nasseroleslami, "The influence of central neuropathic pain in paraplegic patients on performance of a motor imagery based Brain Computer Interface," Clin Neurophysiol. vol. 216, no. 11, pp. 2170-2180, Nov 2015.

53. E. López-Larraz, J.M. Antelis, L, Montesano, A. Gil-Agudo, J. Minguez. "Continuous decoding of motor attempt and motor imagery from EEG activity in spinal cord injury patients." (2012. Aug) Presented at IEEE Eng Med Biol Soc. doi: 10.1109/EMBC.2012.6346299. 